\title{
Tyrosine Kinase Signaling in and on the Endoplasmic Reticulum
}

\author{
Hesso Farhan \\ Institute of Basic Medical Sciences, University of Oslo, Norway \\ Email: hesso.farhan@medisin.uio.no
}

\begin{abstract}
Tyrosine kinases are signaling molecules that are common to all metazoans and are involved in the regulation of many cellular processes such as proliferation and survival. While most attention has been devoted to tyrosine kinases signaling at the plasma membrane and the cytosol, very little attention has been dedicated to signaling at endomembranes. In this review, I will discuss recent evidence that we obtained on signaling of tyrosine kinases at the surface of the endoplasmic reticulum, as well as in the lumen of this organelle. I will discuss how tyrosine kinase signaling might regulate endoplasmic reticulum proteostasis and the implication thereof to general cell physiology.
\end{abstract}


Tyrosine kinases catalyze a very frequently occurring phosphorylation event that is essential for multicellular organisms. The absence of tyrosine kinases in many unicellular organisms led to the view that they are specific to metazoan (1). While many unicellular organisms lack tyrosine kinases, evidence has been obtained that they exist in choanoflagellates (2). Tyrosine kinases occur as two main types: transmembrane receptor tyrosine kinases (RTKs) and soluble tyrosine kinases. The latter may either be cytosolic or they can be found in the lumen of organelles from where they are eventually secreted to the extracellular space. Tyrosine phosphorylation can also be performed by dual-specificity kinases, but the current review will only focus on tyrosine kinases. Several excellent reviews have already discussed tyrosine kinases with respect to their structure, function, their roles in diseases and the possibilities to target them pharmacologically for diseases therapy (3-6). However, the attention has mainly focused on signaling events triggered by RTKs from the cell surface or by roles of cytosolic tyrosine kinases in signaling networks. Much less attention has been dedicated to the spatial signaling of tyrosine kinases on intracellular organelles. Recently, several reports have shown that tyrosine kinases are active in lumen of organelles and on the surface of the endoplasmic reticulum (ER). The ER is the largest organelle of the cell and is responsible for folding and trafficking a third of the eukaryotic proteome. Thus, the ER is a key organelle involved in balancing cellular protein homeostasis (proteostasis) (7). In this review, I will first provide a concise overview of the ER proteostasis network and then discuss its modulation by different tyrosine kinases that are active in and on the ER.

\section{The ER proteostasis network}

A third of the eukaryotic proteome harbors signal sequences that target these proteins to the ER. One of the main functions of the ER is to fold and quality control these proteins. In addition, a cytosolic machinery composed of GTPases, coat proteins SNAREs and tethering proteins is involved in orchestrating trafficking to and from the ER. The balance between protein folding, trafficking and degradation is referred to as ER-proteostasis. The folding machinery is composed of chaperones and enzymes for post-translational protein modification such as glycosylating enzymes or oxidoreductases. After release from the quality control machinery, secretory proteins leave the ER at specialized domains called ER exit sites (ERES), where COPII vesicles are formed that ferry cargo to distal compartments. The COPII assembly cascade starts with activation of the small GTPase Sar1 by its exchange factor Sec1 2 followed by recruitment of the Sec23-Sec24 dimer that captures cargo that is destined for export. The process is completed by recruitment of the Sec13-Sec31 heterotetramer that then helps deform the membrane and facilitates budding of the COPII carrier (8). While ER export was considered long to be a constitutive process, there is mounting evidence that it is subject to regulation by multiple signaling cascades (9-13). Apart from being the first step of a secretory journey, ER export plays another important homeostatic role, namely by unloading the ER and thereby preventing overload of this compartment. The ER can be overloaded either because of a retardation of ER export, or by protein misfolded, which generates export incompetent and sometimes toxic proteins. Such conditions represent a threat to the 
balance of the ER and trigger the unfolded protein response (UPR) that activates three signal transducers (IRE1, ATF6 and PERK) that initiates transcription of genes encoding chaperones as well as the machinery for vesicles trafficking (14).

\section{Receptor Tyrosine Kinases}

Receptor tyrosine kinases (RTKs) are a subclass of tyrosine kinases comprising 58 known members in humans that fall into 20 subfamilies (3). All RTKs share a similar protein structure composed of an extracellular ligand binding domain, a single transmembrane helix, and an intracellular region that contains the tyrosine kinase domain (TKD) as well as other domains involved in protein-protein interactions. This principal topology and the mechanism of action is conserved from nematodes to mammals. In general, RTKs localize to the cell surface, which is expected as they are supposed to bind to extracellular ligands. However, mutant versions of RTKs were reported to localize to intracellular membranes. Internal tandem duplications in the tyrosine kinase receptor FLT3 (FLT3-ITD) are frequently found in acute myeloid leukemia. FLT3-ITD was reported to localize to the ER and to trigger aberrant oncogenic signaling $(15,16)$. Importantly, signaling of FLT3-ITD from the ER was ligandindependent.

Another example for the role of the ER in RTK signaling comes again from cancer. Overexpression of the ErbB3 receptor tyrosine kinase contributes to the promotion of a variety of tumors. Fry et al (17) proposed that nascent ErbB3 is degraded by the ER-localized ubiquitin ligase Nrdp1 and that the loss of the latter in cancer results in ErbB3 overexpression. Thus, spatial regulation of protein degradation at the ER contributes to regulation of ErbB3 signaling.

The aforementioned examples are cases that support a role of the ER in RTK signaling under pathologic conditions. However, there is also evidence that the ER contributes top RTK signaling under physiologic conditions. For instance, ER-localized protein tyrosine phosphatase PTP1B plays an important role in dephosphorylating and inactivating active RTKs after internalization $(18,19)$. This takes place on endosomes carrying RTKs that make contacts with the ER. Thus, the ER contributes to RTK signaling by providing a platform for their deactivation. Nevertheless, signaling of normal (i.e. non-mutant or oncogenic) RTKs from the ER remained elusive and was considered unlikely, because no RTK ligands are present at this location. Thus, it came as a surprise to find that the receptor tyrosine kinase LTK (Leukocyte Tyrosine Kinase) localizes to the general ER and in some cases also to ER exit sites (20). LTK forms a subfamily together with the anaplastic lymphoma kinase (ALK), with which it shares strong sequence similarity in the kinase domain. However, the luminal/extracellular domains are different in mammals, as LTK lacks a large portion that is otherwise present in ALK. LTK homologs from non-mammalian species such as birds of fish do not show this difference between ALK and LTK (20). This shows that mammalian LTK is different from that in other species. ER localization of LTK has been suggested previously for overexpressed LTK (21), but this finding always suffered from the fact that many RTKs localize to intracellular compartments when overexpressed. Our finding that 
endogenous LTK localizes to and is active at the ER were therefore unexpected. Moreover, LTK appeared to be trapped in the ER and showed no signs of ever leaving this location, implying that it regulates biologic processes at the ER. When mapping the LTK interactome, Sec12 was identified as one of the binding partners, suggesting a role for regulation of secretory trafficking. Indeed, LTK phosphorylates Sec12 and thereby regulates export of secretory proteins from the ER by stimulating the biogenesis of ER exit sites (20). While these findings suggest that LTK might be a regulator of proteostasis, this is far from being understood as it is unclear for instance what signal triggers the activation of LTK. Recent findings proposed that LTK is a receptor for secreted ligands FAM150A\&B (also referred to as ALKAL1\&2) (22-25). It is hard to reconcile our findings that LTK is resident to the ER with its activation by an extracellular ligand. However, one key difference is that our work was carried out in cells that do not have the sister protein ALK, while the others were carried out in cells that are ALK/LTK double positive. Certainly, more work is needed to elucidate the mechanisms and regulators of LTK activation. A hallmark of RTKs is the formation of dimers or larger-order oligomers. These oligomers are either constitutive as in the case of insulin receptor or are ligand induced as with the majority of known cases $(3,26)$. LTK is distantly related to the insulin receptor, and thus it might form ligand-independent oligomers. Whether this is true remains to be shown.

\section{Luminal Tyrosine Kinases}

When analyzing the mammalian phosphoproteome, a surprising finding was that a large fraction of proteins secreted to the extracellular space are phosphorylated, including on tyrosine residues (27). This pointed to the existence of a secreted tyrosine kinase that performs all the phosphorylation events outside the cell, or alternatively, to a kinase that localizes to the lumen of the secretory pathway and phosphorylates secretome components prior to their secretion. Secretome components (such as casein or osteopontin) were already known for many decades to be phosphorylated on serine residues, but it was only in 2012 that FAM20C was identified as the kinase responsible for these phosphorylation events (28). Only two years later, VLK (also known as PKDCC or SGK493) was identified as the secreted tyrosine kinase (29). VLK contains a signal peptide that mediates its entry into the lumen of the ER. Besides being active outside the cell, VLK appears to phosphorylate proteins already in the ER. Strikingly, among the phosphorylated proteins are components of the ER-proteostasis machinery such as ERP29, ERP44, BiP and PDIA3. It is however possible that these proteins leak out of the ER and are secreted outside the cell, where they are phosphorylated and more work is needed to identify the site of phosphorylation. The catalytic activity of VLK was important for its secretion, tempting to speculate that VLK regulates its own trafficking by phosphorylating a trafficking receptor. It is likely that VLK relies on a transmembrane cargo receptor for exiting the ER, because is a soluble luminal protein and has no other possibility to interact with the cytosolic COPII machinery that mediates ER export. Whether 
this assumption is true remains to be determined, but because VLK is a glycoprotein, it is possible that its transport is regulated by an ER lectin.

It is currently unclear what the biologic role of the phosphorylation of ER-resident proteins is. It is also unknown whether VLK activity in the ER is constitutive or whether it is subject to regulation, for instance by fluctuation of the ionic or $\mathrm{pH}$ balance of the ER. It might be possible that the phosphorylation of ER chaperones functionally regulates these proteins and thereby has an impact on the folding capacity of the ER. This opens the possibility to test the role of VLK in the regulation of ER-proteostasis and represents and exciting future research direction.

The identification of VLK as a luminal tyrosine kinase raises the question of whether other tyrosine kinases exist in the lumen of the ER (or other organelles). Furthermore, we might speculate whether VLK itself phosphorylates more proteostasis-relevant substrates that have been missed in the original study. Support for the existence of other luminal tyrosine kinases comes from inspecting publicly available databases. Based on the Phosphosite plus website several ER proteins are tyrosine phosphorylated. In table 1, I have listed a manually extracted collection of phospho-tyrosine residues on regulators of ER proteostasis (e.g. chaperones or cargo receptors). This illustrates that tyrosine phosphorylation is not restricted to a handful of ER proteins and that more roles for this posttranslational modification will be identified in the future. In particular, BiP and calreticulin appear to be heavily tyrosine phosphorylated, implying that these post-translational modifications are likely to be functionally relevant (table 1). The identification of new luminal tyrosine kinases represents an exciting area for future investigations. Potential candidates for this are members of the FAM69 subfamily, which were shown to localize to the ER (30) and that are related (based on sequence) to VLK and are predicted to act as kinases (31).

\section{Cytosolic Tyrosine Kinases}

Several cytosolic tyrosine kinases are known to localize to endomembranes, but research has focused mainly on the Golgi apparatus or the endolysosomal system. Less is known about cytosolic tyrosine kinases that localize to, and signal at the ER. One of the first examples was the phosphorylation of IRE1 $\alpha$ by the cytosolic tyrosine kinase ABL. IRE1 $\alpha$ is an ER-resident transmembrane kinase with endoribonuclease (RNase) activity and is one of the three main transducers of the unfolded protein response (UPR) and ER stress. Upon accumulation of misfolded proteins in the ER, IRE1 $\alpha$ oligomerizes and undergoes trans-autophosphorylation, leading to activation of the RNase domain and initiating frame-shift splicing of the mRNA encoding XBP1 transcription factor (32). This triggers adaptive UPR that ensures that cells remain fit under stressful conditions. In case the ER stressor persists, IRE1 $\alpha$ organizes into high-order oligomers and RNase activation state rise further, thereby causing degradation of many ER-localized mRNAs and apoptosis (33-35). This ER-stress induced apoptosis is observed in several diseases with pre-mature cell loss such as in pancreatic islet $\beta$-cells under diabetic conditions $(36,37)$. The cytosolic tyrosine kinase ABL was found to localize to the ER membrane, where it 
hyperactivates IRE1 $\alpha$. This increases the RNase activity and thereby potentiates ER stress-induced apoptosis (38). Because kinases are druggable, this offered the possibility to modulate the apoptosis associated with ER stress. Accordingly, Imatinib which blocks ABL activity, was shown to prevent loss of $\beta$-cells in diabetic mice (38). How exactly ABL is activated by ER stress is not completely understood, but nevertheless, inhibition of ABL could be used therapeutically to prevent the loss of $\beta$-cells due to secretory exhaustion. In support of this, it was observed that patients that are treated with imatinib exhibited lower fasting glucose levels, indicative of an anti-diabetic effect of imatinib (39).

Another tyrosine kinase that was shown to localize to the ER is Src, which acts downstream of oncogenic Ras to promote cancer cell. Invasion, although it was not clearly shown whether the ER pools of Src is responsible for this effect (40). While ABL acted upstream of IRE1 $\alpha$, Src family kinases appear to be downstream (41). Activation of Src by IRE1 $\alpha$ resulted in activation of Arf GTPase at the Golgi and reduced retrograde trafficking from the Golgi back to the ER. Consequently, retention of ER chaperones was weaker resulting in their secretion outside cells. Extracellular deposition of ER chaperones is a widely known observation in many cancers although we still do not fully understand the pathophysiological relevance of this phenomenon, although this is generally considered a protumorigenic effect. For instance, localization of BiP/GRP78 to the cell surface leads to an alteration of the tumor-suppressive effects of TGF $\beta$ (42). Because Src family kinases play a role in many malignant diseases, it is tempting to speculate that part of the pro-tumorigenic effect of these kinases is to facilitate the secretion of ER chaperones.

Another notable tyrosine phosphorylation event at the ER is the phosphorylation of the ER stress transducer PERK. Upon induction of ER stress, PERK becomes phosphorylated on Y619 within its kinase domain (43). Although PERK is a Ser/Thr kinase, it was proposed that it also possesses tyrosine kinase activity (making it a dual specificity kinase) and that Y619 is phosphorylated in trans. This phosphorylation is important for the regulation of PERK activity, because the Y619F mutant of PERK failed to induce translation of ATF4. This tyrosine residue is dephosphorylated by the ER-localized phosphatase PTP1B, and accordingly, inhibition of this phosphatase results in higher ER stress (44). PTP1B was also earlier shown to dephosphorylate IRE1 and to potentiate its signaling (45).

PERK was also reported to be phosphorylated on Y561 in the juxtamembrane region (46), which creates a binding site for the adaptor protein Nck1, which reduces PERK activity. Furthermore, Nck1 binding to PERK was demonstrated to modulate the effect of PERK on insulin production and accordingly, Nck1 depleted cells or mice produced more insulin. How exactly Nck1 negative regulates PERK is unclear, but one possibility is that it hinders the formation of PERK dimers.

\section{Perspectives}

(i) Tyrosine kinase signaling in and on the ER is an emerging area of research that has strong potential to expand our understanding of the regulation of ER proteostasis (Figure 1) 
(ii) We know at the moment only a handful of tyrosine kinases operating in and on the ER. In particular, there is strong potential for the discovery of new tyrosine kinases that are active in the ER lumen

(iii) Future work will need to focus on one hand on discovering new tyrosine kinases but on the other hand stronger emphasis must be devoted to uncover upstream signals that trigger these kinases as for instance neither VLK nor LTK have any known upstream regulators.

(iv) The signaling toolkit for tyrosine phosphorylation is composed of the kinase, the phosphatase and adaptor molecules recognizing the phosphotyrosine. We do not know the entire signaling toolkit for any of the signaling events discussed in this review. Thus, future work must focus on identifying all elements of a tyrosine kinase toolkit.

\section{References}

1. Hunter T, Cooper JA. Protein-tyrosine kinases. Annu Rev Biochem. 1985;54:897-930.

2. King $\mathrm{N}$, Hittinger CT, Carroll SB. Evolution of key cell signaling and adhesion protein families predates animal origins. Science. 2003;301(5631):361-3.

3. Lemmon MA, Schlessinger J. Cell signaling by receptor tyrosine kinases. Cell. 2010;141(7):1117-34.

4. Du Z, Lovly CM. Mechanisms of receptor tyrosine kinase activation in cancer. Mol Cancer. 2018;17(1):58-.

5. Krause DS, Van Etten RA. Tyrosine kinases as targets for cancer therapy. N Engl J Med. 2005;353(2):172-87.

6. Rivera-Torres J, San José E. Src Tyrosine Kinase Inhibitors: New Perspectives on Their Immune, Antiviral, and Senotherapeutic Potential. Front Pharmacol. 2019;10:1011-.

7. Klaips CL, Jayaraj GG, Hartl FU. Pathways of cellular proteostasis in aging and disease. J Cell Biol. 2018;217(1):51-63.

8. Peotter J, Kasberg W, Pustova I, Audhya A. COPII-mediated trafficking at the ER/ERGIC interface. Traffic. 2019;20(7):491-503.

9. Centonze FG, Farhan H. Crosstalk of endoplasmic reticulum exit sites and cellular signaling. FEBS Lett. 2019;593(17):2280-8.

10. Zacharogianni M, Kondylis V, Tang $\mathrm{Y}$, Farhan $\mathrm{H}$, Xanthakis $\mathrm{D}$, Fuchs $\mathrm{F}$, et al. ERK7 is a negative regulator of protein secretion in response to amino-acid starvation by modulating Sec16 membrane association. EMBO J. 2011;30(18):3684-700.

11. Tillmann KD, Millarte $V$, Farhan $H$. Regulation of traffic and organelle architecture of the ER-Golgi interface by signal transduction. Histochem Cell Biol. 2013;140(3):297-306.

12. Farhan $H$, Wendeler MW, Mitrovic S, Fava E, Silberberg $Y$, Sharan $R$, et al. MAPK signaling to the early secretory pathway revealed by kinase/phosphatase functional screening. J Cell Biol. 2010;189(6):997-1011. 
13. Farhan $\mathrm{H}$, Rabouille $\mathrm{C}$. Signalling to and from the secretory pathway. J Cell Sci. 2011;124(Pt 2):171-80.

14. Walter $P$, Ron $D$. The unfolded protein response: from stress pathway to homeostatic regulation. Science. 2011;334(6059):1081-6.

15. Schmidt-Arras D, Böhmer S-A, Koch S, Müller JP, Blei L, Cornils H, et al. Anchoring of FLT3 in the endoplasmic reticulum alters signaling quality. Blood. 2009;113(15):3568-76.

16. Choudhary C, Olsen JV, Brandts C, Cox J, Reddy PNG, Böhmer FD, et al. Mislocalized activation of oncogenic RTKs switches downstream signaling outcomes. Mol Cell. 2009;36(2):326-39.

17. Fry WHD, Simion C, Sweeney C, Carraway KL, 3rd. Quantity control of the ErbB3 receptor tyrosine kinase at the endoplasmic reticulum. Mol Cell Biol. 2011;31(14):3009-18.

18. Stuible M, Tremblay ML. In control at the ER: PTP1B and the down-regulation of RTKs by dephosphorylation and endocytosis. Trends Cell Biol. 2010;20(11):672-9.

19. Sangwan V, Abella J, Lai A, Bertos N, Stuible M, Tremblay ML, et al. Protein-tyrosine phosphatase $1 \mathrm{~B}$ modulates early endosome fusion and trafficking of Met and epidermal growth factor receptors. J Biol Chem. 2011;286(52):45000-13.

20. Centonze FG, Reiterer V, Nalbach K, Saito K, Pawlowski K, Behrends C, et al. LTK is an ER-resident receptor tyrosine kinase that regulates secretion. J Cell Biol. 2019;218(8):247080.

21. Bauskin AR, Alkalay I, Ben-Neriah Y. Redox regulation of a protein tyrosine kinase in the endoplasmic reticulum. Cell. 1991;66(4):685-96.

22. Zhang H, Pao LI, Zhou A, Brace AD, Halenbeck R, Hsu AW, et al. Deorphanization of the human leukocyte tyrosine kinase (LTK) receptor by a signaling screen of the extracellular proteome. Proc Natl Acad Sci U S A. 2014;111(44):15741-5.

23. Reshetnyak AV, Murray PB, Shi X, Mo ES, Mohanty J, Tome F, et al. Augmentor $\alpha$ and $\beta$ (FAM150) are ligands of the receptor tyrosine kinases ALK and LTK: Hierarchy and specificity of ligand-receptor interactions. Proc Natl Acad Sci U S A. 2015;112(52):15862-7.

24. Guan J, Umapathy G, Yamazaki Y, Wolfstetter G, Mendoza P, Pfeifer K, et al. FAM150A and FAM150B are activating ligands for anaplastic lymphoma kinase. Elife. 2015;4:e09811-e.

25. Fadeev A, Mendoza-Garcia P, Irion U, Guan J, Pfeifer K, Wiessner S, et al. ALKALs are in vivo ligands for ALK family receptor tyrosine kinases in the neural crest and derived cells. Proc Natl Acad Sci U S A. 2018;115(4):E630-E8.

26. Ward CW, Lawrence MC, Streltsov VA, Adams TE, McKern NM. The insulin and EGF receptor structures: new insights into ligand-induced receptor activation. Trends Biochem Sci. 2007;32(3):129-37.

27. Hornbeck PV, Chabra I, Kornhauser JM, Skrzypek E, Zhang B. PhosphoSite: A bioinformatics resource dedicated to physiological protein phosphorylation. Proteomics. 2004;4(6):1551-61.

28. Tagliabracci VS, Engel JL, Wen J, Wiley SE, Worby CA, Kinch LN, et al. Secreted kinase phosphorylates extracellular proteins that regulate biomineralization. Science. 2012;336(6085):1150-3.

29. Bordoli MR, Yum J, Breitkopf SB, Thon JN, Italiano JE, Jr., Xiao J, et al. A secreted tyrosine kinase acts in the extracellular environment. Cell. 2014;158(5):1033-44.

30. Tennant-Eyles AJ, Moffitt H, Whitehouse CA, Roberts RG. Characterisation of the FAM69 family of cysteine-rich endoplasmic reticulum proteins. Biochem Biophys Res Commun. 2011;406(3):471-7. 
31. Dudkiewicz $M$, Lenart A, Pawłowski K. A novel predicted calcium-regulated kinase family implicated in neurological disorders. PLoS One. 2013;8(6):e66427-e.

32. Yoshida H, Matsui T, Yamamoto A, Okada T, Mori K. XBP1 mRNA is induced by ATF6 and spliced by IRE1 in response to ER stress to produce a highly active transcription factor. Cell. 2001;107(7):881-91.

33. Han D, Lerner AG, Vande Walle L, Upton J-P, Xu W, Hagen A, et al. IRE1alpha kinase activation modes control alternate endoribonuclease outputs to determine divergent cell fates. Cell. 2009;138(3):562-75.

34. Aragón $T$, van Anken E, Pincus D, Serafimova IM, Korennykh AV, Rubio CA, et al. Messenger RNA targeting to endoplasmic reticulum stress signalling sites. Nature. 2009;457(7230):736-40.

35. Shamu CE, Walter P. Oligomerization and phosphorylation of the Ire1p kinase during intracellular signaling from the endoplasmic reticulum to the nucleus. EMBO J. 1996;15(12):3028-39.

36. Oakes SA, Papa FR. The role of endoplasmic reticulum stress in human pathology. Annu Rev Pathol. 2015;10:173-94.

37. Scheuner D, Kaufman RJ. The unfolded protein response: a pathway that links insulin demand with beta-cell failure and diabetes. Endocr Rev. 2008;29(3):317-33.

38. Morita S, Villalta SA, Feldman HC, Register AC, Rosenthal W, Hoffmann-Petersen IT, et al. Targeting ABL-IRE1 $\alpha$ Signaling Spares ER-Stressed Pancreatic $\beta$ Cells to Reverse Autoimmune Diabetes. Cell Metab. 2017;25(5):1207-.

39. Gómez-Sámano MÁ, Baquerizo-Burgos JE, Coronel MFC, Wong-Campoverde BD, Villanueva-Martinez F, Molina-Botello $D$, et al. Effect of imatinib on plasma glucose concentration in subjects with chronic myeloid leukemia and gastrointestinal stromal tumor. BMC Endocr Disord. 2018;18(1):77-.

40. Chan P-C, Chen H-C. p120RasGAP-mediated activation of c-Src is critical for oncogenic Ras to induce tumor invasion. Cancer Res. 2012;72(9):2405-15.

41. Tsai Y-L, Ha DP, Zhao H, Carlos AJ, Wei S, Pun TK, et al. Endoplasmic reticulum stress activates SRC, relocating chaperones to the cell surface where GRP78/CD109 blocks TGF- $\beta$ signaling. Proc Natl Acad Sci U S A. 2018;115(18):E4245-E54.

42. Wiersma VR, Michalak M, Abdullah TM, Bremer E, Eggleton P. Mechanisms of Translocation of ER Chaperones to the Cell Surface and Immunomodulatory Roles in Cancer and Autoimmunity. Front Oncol. 2015;5:7-.

43. Su Q, Wang S, Gao HQ, Kazemi S, Harding HP, Ron D, et al. Modulation of the eukaryotic initiation factor 2 alpha-subunit kinase PERK by tyrosine phosphorylation. J Biol Chem. 2008;283(1):469-75.

44. Krishnan N, Fu C, Pappin DJ, Tonks NK. H2S-Induced sulfhydration of the phosphatase PTP1B and its role in the endoplasmic reticulum stress response. Sci Signal. 2011;4(203):ra86ra.

45. Gu F, Nguyên DT, Stuible M, Dubé N, Tremblay ML, Chevet E. Protein-tyrosine phosphatase $1 \mathrm{~B}$ potentiates IRE1 signaling during endoplasmic reticulum stress. J Biol Chem. 2004;279(48):49689-93.

46. Yamani L, Latreille M, Larose L. Interaction of Nck1 and PERK phosphorylated at $Y^{561}$ negatively modulates PERK activity and PERK regulation of pancreatic $\beta$-cell proinsulin content. Mol Biol Cell. 2014;25(5):702-11. 


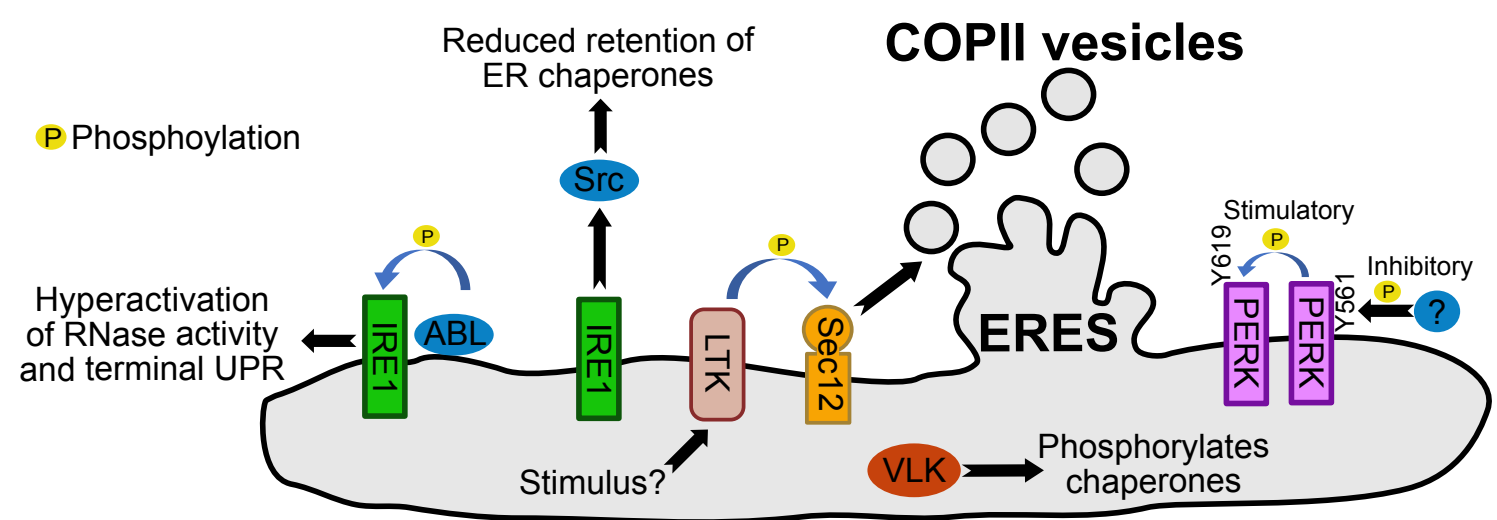

ENDOPLASMIC RETICULUM

Figure 1. Schematic representation of tyrosine kinases signaling in and on the ER as described in the text.

Table 1. List of ER luminal proteins and known phosphotyrosine residues on them

\begin{tabular}{|l|l|}
\hline \multicolumn{1}{|c|}{ ER proteostasis regulator } & \multicolumn{1}{|c|}{ Phosphorylated tyrosine residues } \\
\hline BiP (HSPA5) & $\begin{array}{l}\text { Y39, Y65, Y127, Y160, Y175, Y209, Y313, } \\
\text { Y466, Y568, Y570, Y653 }\end{array}$ \\
\hline VIP36 & Y252, Y251 \\
\hline ERGIC53 & Y430 \\
\hline Calnexin & Y70, Y185, Y214, Y379, Y393, Y514 \\
\hline Calreticulin & $\begin{array}{l}\text { Y22, Y57, Y75, Y109, Y150, Y172, Y182, Y271, } \\
\text { Y285, Y299 }\end{array}$ \\
\hline BAP31 & Y73 \\
\hline PDIA3 & $\begin{array}{l}\text { Y67, Y95, Y100, Y115, Y196, Y222, Y269, } \\
\text { Y278, Y365, Y445, Y454 }\end{array}$ \\
\hline ERO1L & Y73, Y248, Y266, Y431 \\
\hline
\end{tabular}

